\title{
Time and Frequency Domain Channel Estimation in MIMO-OFDM Co-operative Communication
}

\author{
Jancy Wilson ${ }^{1}$, Aravind S. ${ }^{2}$ \\ ${ }^{1}$ P. G. Scholar, Department of Communication Engineering, Sree Buddha College of Engineering for Women, Elavumthitta, Kerala, India \\ ${ }^{2}$ Assistant Professor, Department of Electronics and Communication Engineering, Sree Buddha College of Engineering for Women, \\ Elavumthitta, Kerala, India
}

\begin{abstract}
MIMO-OFDM is a promising technique to achieve reliable communication in wireless communication systems. MIMOOFDM system is incorporated with co-operative communication which increases the transmission range. Multiple input multiple output systems (MIMO) divides the input data into different data blocks and is allowed to transmit through multiple antennas. Orthogonal frequency division multiplexing (OFDM) has the ability to cope up severe channel conditions. Multiple transmit and receive antennas in OFDM systems can improve communication quality and capacity. This paper develops a low complexity channel estimation in MIMO-OFDM communication. The priori-information aided iterative hard threshold algorithm (PAIHT) is the channel estimation technique which uses prior information for obtaining accurate channel estimate of the wireless channel. Simulation results demonstrate that, the proposed scheme performs well without sacrificing spectral efficiency.
\end{abstract}

Keywords: Multiple input multiple output (MIMO), Orthogonal frequency division multiplexing (OFDM), Priori-information aided iterative hard threshold algorithm (PAIHT)

\section{Introduction}

The idea of space time block codes has been applied in wireless relay networks in order to obtain the benefits of multiple input multiple output (MIMO) systems. Multiple input multiple output technique uses multiple antennas at both transmitting and receiving end. The signals are transmitted using multiple antennas from the transmitting end. Spatial multiplexing requires MIMO antenna configuration. In spatial multiplexing, a high-rate signal is split into multiple lower-rate streams and each stream is transmitted from a different transmit antenna in the same frequency channel. If these signals arrive at the receiver antenna array with sufficiently different spatial signatures and the receiver has accurate channel state information, it can separate these streams into (almost) parallel channels. Spatial multiplexing is a very powerful technique for increasing channel capacity at higher signal-to-noise ratios (SNR). Given multiple antennas, the spatial dimension can be exploited to improve the performance of a wireless link.

Orthogonal frequency-division multiplexing (OFDM) technology has been widely applied in high-speed broadband wireless communication systems. Orthogonal frequencydivision multiplexing (OFDM) provides an effective and low complexity technique which eliminates inter symbol interference for transmission over frequency selective fading channels. The problems created by multipath fading effects are efficiently handled by using MIMO-OFDM technique. The main advantage of MIMO-OFDM technique is the ability to cope up with severe channel conditions. MIMOOFDM can achieve high spectral efficiency even when the transmitter does not possess any channel state information. When the transmitter posses channel state information, it is possible to approach theoretical capacity.
Channel estimation is to estimate the filter coefficient through received signal and other known information. Prioriinformation aided iterative hard threshold algorithm (PAIHT) utilizes the priori-information of the acquired coarse estimate. This algorithm is based on compressed sensing technique. The priori information of the acquired coarse estimate for the wireless channel is used to obtain an accurate channel estimate of the doubly selective fading channel.

Co-operative relaying, even with a single available relay, can significantly improve the performance of the system. Relays are suitably placed in between source and destination. The amplify-and-forward strategy allows the relay station to amplify the received signal from the source node and to forward it to the destination station. The transmission distance of the communication system can be increased by the introduction of relays.

\section{System Model}

\subsection{Transmitter Section}

In the transmitter section, the data blocks are transmitted on orthogonal subcarriers through parallel antennas. The data is first converted into space time block codes. The space time block codes (STBC) are mapped using any modulation technique. Quadrature amplitude modulation (QAM) is used here as the modulation technique. The space time block codes are modulated on orthogonal subcarriers. The orthogonal subcarriers allows secure communication of the data and also protects from the multipath effects. The mapped space time block codes are converted into the frequency domain and modulated using orthogonal subcarriers. Thus the OFDM signals are transmitted through 


\section{International Journal of Science and Research (IJSR) \\ ISSN (Online): 2319-7064}

Index Copernicus Value (2013): 6.14 | Impact Factor (2015): 6.391

multiple transmitting antennas.

In the time domain, OFDM signals are grouped in symbols, and each OFDM symbol consists of a pseudo-noise (PN) sequence of length $\mathrm{M}$, and the following OFDM data block of length L. At the receiver, the received $i$ th OFDM symbol can be written as $r_{i}=s_{i} * h_{i}+n_{i}$ where $n_{i}$ is the channel additive white Gaussian noise (AWGN) vector having a zero mean and $h_{i}$ is the time-varying channel impulse response (CIR) of length $\mathrm{L}$.

$$
h_{i, l}=\sum_{p=0}^{P-1} \alpha_{i, p} \delta\left(l-\tau_{i, p}\right), 0 \leq l \leq L-1 \text {, }
$$

Where $\alpha_{\mathrm{i}, \mathrm{p}} p$ th path gain and $\tau_{\mathrm{i}, \mathrm{p}}$ is the $p$ th path delay.

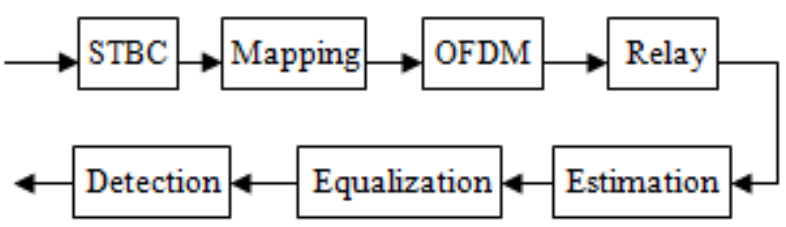

Figure 1: System model

\subsection{Relaying}

The mobile radio environment is characterized by fading effects which cause attenuation or even outage of particular links between wireless communication pairs. Cooperative diversity is a cooperative multiple antenna technique for improving or maximising total network channel capacities for any given set of bandwidths. Relays are placed suitably in between transmitter and receiver. Relays captures the transmitted signal from the source and forwards the signal to the destination. The amplify-and-forward strategy allows the relay station to amplify the received signal from the source node and to forward it to the destination station.

The transmission range can be extended by relaying technique. The power and quality of the signal can be increased. Relaying improves reliability and data throughput. Probability of error can be reduced by processing multiple independent copies of symbol. Relays can be used in improving network connectivity and enhancing power. The spectral efficiency and communication reliability can be increased by the introduction of relays in communication networks.

Relay nodes do not perform any channel estimation using the training symbols transmitted by the source. Relay nodes captures the incoming signal, amplifies the captured signals and forwards these signals to the destination. Thus transmission distance can be increased by the help of relay nodes. The distance between source and destination can be increased with the aid of relay nodes.

\subsection{Channel Estimation}

The channel effects like a filter. Channel estimation is to estimate the filter coefficient through received signal and other known information. The coefficients of channel gives the characteristics of the channel. These coefficients can be easily extracted with the help of an estimation technique. Priori-information aided iterative hard threshold algorithm is an effective channel estimation tool without the loss of spectral efficiency.

\subsubsection{PAIHT}

Priori-information aided iterative hard threshold algorithm (PAIHT) is a low complexity high-accuracy compressed sensing based channel estimation scheme for OFDM systems. The overlap-add method is used to obtain the coarse estimates of the channel length, path delays and path gains of the wireless channel, whereby the temporal correlation of the multipath delays and multipath gains among several consecutive OFDM symbols is exploited. Temporal correlation refers to the similarity of one signal over time.

In this method, $\mathrm{t}$ he tail part caused by the multipath channel is superposed on the preceding main part, and then this overlap-add result is circularly correlated with the local PN sequence to obtain some coarse channel state information (CSI). Moreover, the temporal correlation of the multipath delays and gains among several consecutive OFDM symbols is jointly exploited to improve the robustness and accuracy of the coarse channel estimate.

The proposed channel estimation (CE) method consists of four steps. To be more specific, in the first step, a coarse channel impulse response length and path delays are estimated, while in the second step, coarse channel gains are obtained. With the aid of the coarse information of the wireless channel obtained in the first two steps, the proposed PA-IHT algorithm estimates the accurate path delays in the third step, and finally at the fourth step, the accurate path gains are obtained based on a maximum likelihood (ML) criterion.

Compressed sensing theory helps in the reconstruction of sparse signal even if with the available few observations. Thus with the help of low dimensional uncorrelated observations, the high dimensional signal can be easily reconstructed. The wireless channel is sparse in nature and the actual number of resolvable paths are very much less than channel impulse response length.

\subsubsection{Algorithm}

Step 1: Acquisition of Coarse Channel Length and Path Delays

For the acquisition of coarse channel length and path delays an overlap add method is needed. The PN sequence is termed as the TS main part and a portion of the OFDM data is termed as TS tail part. Average the absolute value of timevarying channel impulse response among continuous OFDM symbol. Figure 2 represents overlap-add method of the $i$ th OFDM signal.

$$
\mathbf{r}_{k}=\mathbf{r}_{k, \text { main }}+\mathbf{r}_{k, \text { tail }}, i-R_{d}+1 \leq k \leq i+R_{d} \text {, }
$$

Where $\mathbf{r}_{k, \text { main }}$ is the TS main part and $\mathbf{r}_{k \text {, tail }}$ is the TS tail part. 


\section{International Journal of Science and Research (IJSR) \\ ISSN (Online): 2319-7064}

Index Copernicus Value (2013): 6.14 | Impact Factor (2015): 6.391

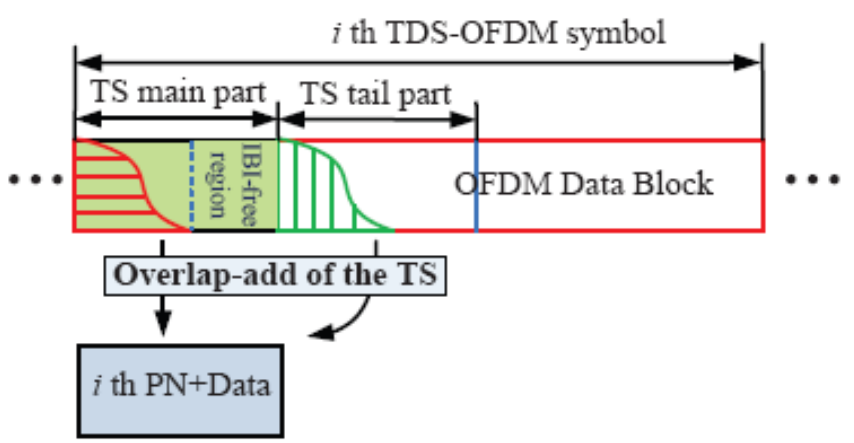

Figure 2: Illustration for overlap-add of the TS in the $i$ th OFDM symbol.

Step 2: Acquisition of Coarse Channel Path Gains

In the second step, averaging of the adjacent PN sequence and data takes place. The result is circularly correlated with locally generated PN sequence. The coarse estimates of the channel length, the channel path delays and path gains acquired in Steps 1 and 2 provide the priori information of the wireless channel to assist the accurate channel estimation using the PA-IHT algorithm in the following two steps.

$$
\overline{\mathbf{h}}^{\prime}=\mathbf{c} \otimes \frac{1}{2 R_{g 2} M} \sum_{k=i-R_{g 2}+1}^{i+R_{g 2}}\left(\mathbf{r}_{k, \text { main }}+\mathbf{r}_{k, \text { tail }}^{\prime}\right)
$$

Step 3: Acquisition of Accurate Path Delay Estimate To improve signal recovery accuracy and to reduce the computational complexity priori-information aided iterative hard threshold algorithm uses the priori information of the coarse channel estimate. The PA-IHT algorithm exploits the available priori information of the coarse path delays and gains as the initial condition, and this significantly enhance the signal recovery accuracy and reduces the number of required iterations. The sizes of the IBI-free region and the measurement matrix are adaptively determined by the coarse channel length estimate. The coarse path gains serve as the nonzero element values of the target signal in every iteration.

$$
\overline{\mathbf{y}}=\frac{1}{2 R_{g 2}} \sum_{k=i-R_{g 2}+1}^{i+R_{g 2}} \mathbf{y}_{k},
$$

Step 4: Accurate Path Gain Estimation

Accurate Path Gain Estimation is based on ML estimation. Maximum likelihood estimation can be used for accurate path delay. Mean square estimation can also be used.

\subsection{Receiver Section}

The coefficients of channel are extracted by the channel estimation technique. The multipath fading effects can be easily studied by the help of channel estimate. If the channel is a having severe channel conditions, then the data received will be highly contaminated. The training sequence added in front of the OFDM signal helps in estimating the channel conditions. Proper equalization can be done after the channel estimation.
The original data can be retrieved by demodulating the received signal. The effect of additive white gaussian noise is removed from the received data. Using training sequence the effects of the channel can be properly studied. Equalization can be done if the data is severely contaminated. If the channel is not such a fading channel, equalization can be avoided. The subcarriers are removed from the OFDM data. Proper detection have to be done to collect the original data. Demodulation is the last step in the receiving section. The demodulated data will be same as the transmitted one.

\section{Simulation Results}

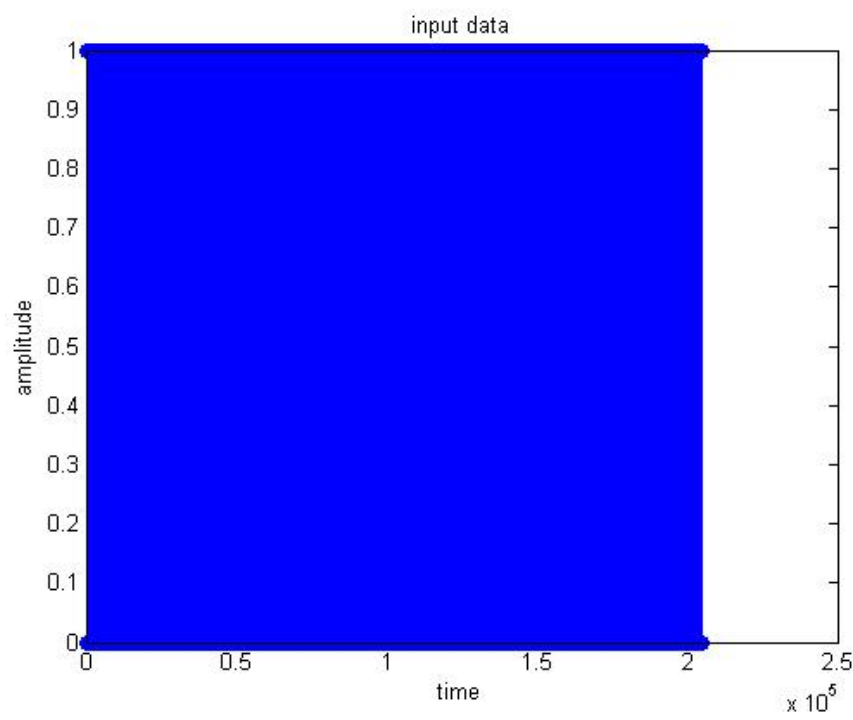

Figure 3: Input data

Mat lab R2013a is used as the platform to perform this task. Figure 3 shows the input signal. The input signal is then divided into number of blocks. The block of data is allowed for modulation. The mapped data will undergo subcarrier modulation. Thus, the signals are transmitted as OFDM signals.

Relay nodes captures the transmitted signals from the source and amplifies the same signal. The amplified signals are forwarded to the destination. Thus the relays just captures the OFDM signal, amplifies it and forward it to the destination. Figure 4 shows the output of relay node.

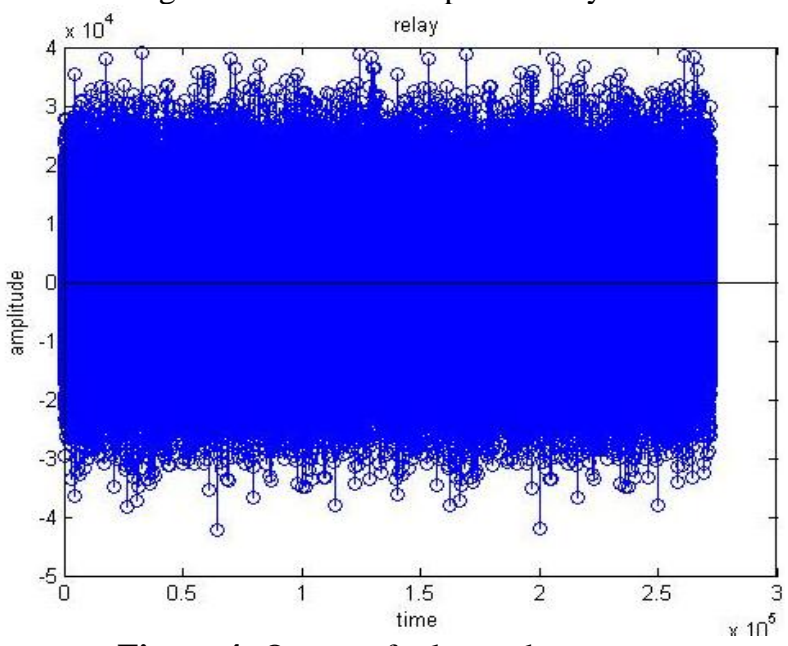

Figure 4: Output of relay node 


\section{International Journal of Science and Research (IJSR) \\ ISSN (Online): 2319-7064}

Index Copernicus Value (2013): 6.14 | Impact Factor (2015): 6.391

At the receiver side, first process is noise removal followed by channel estimation. The training sequence placed before

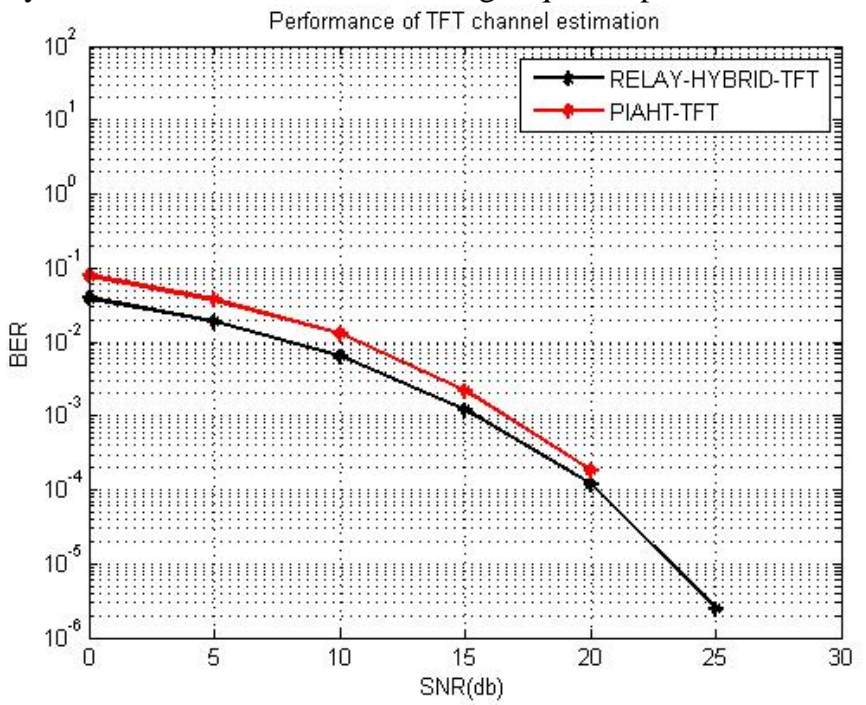

Figure 5: Performance of channel estimation using ML criteria

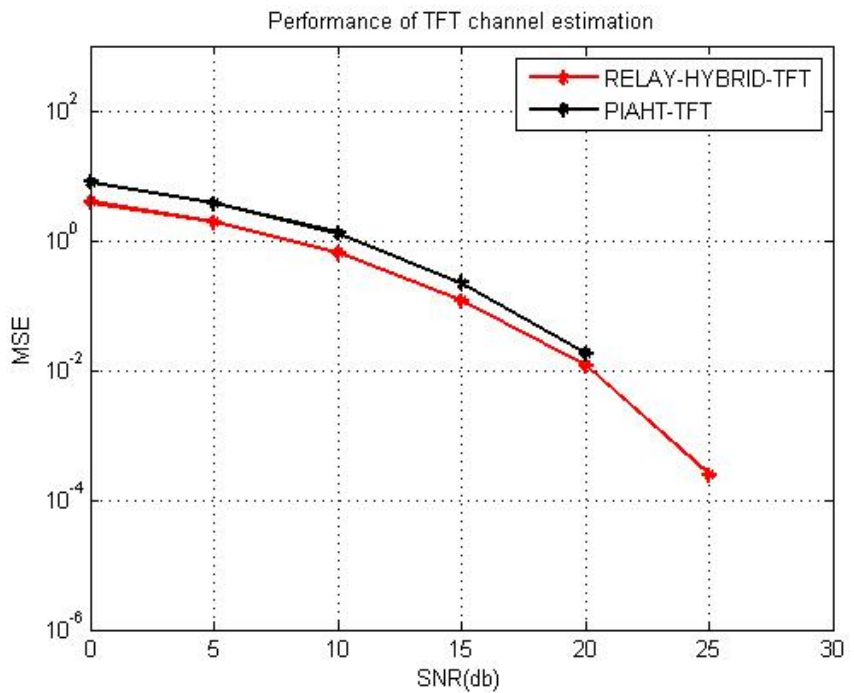

Figure 6: Performance of channel estimation using MSE criteria

the OFDM data is used to estimate the channel. Prioriinformation aided iterative hard threshold algorithm utilizes the priori information to extract the channel information. Thus the characteristics of the channel can be easily studied by estimating the channel. Figure 5 shows the channel estimation using maximum likelihood criteria.

Priori-information aided iterative hard threshold algorithm estimates the channel coefficients without the loss of any spectral efficiency. Figure 6 shows the channel estimation using minimum error criteria.

\section{Conclusion}

Reliable communication in wireless systems can be easily achieved by the implementation of MIMO - OFDM technique. Co-operative communication introduces relay nodes in between source and destination. Thus the transmission distance and quality of the signal can be increased. The multipath fading effects is the main issue in wireless communication. Channel estimation helps in estimating the effects of the channel. Thus by proper channel estimation, the distortions happened in the data can be easily detected. Equalization can be done if data is affected by severe channel conditions. Priori-information aided iterative hard threshold algorithm utilizes the priori information of the OFDM signal to find out the channel estimate. Thus without the loss of spectral efficiency proper channel estimation can be done using (PAIHT).

\section{Acknowledgment}

I would like to express profound gratitude to our Head of the Department, Prof. Cherian Schariah, for his encouragement and for providing all facilities for my work. I express my highest regard and sincere thanks to my guide, Asst. Prof. Mr. Aravind S., who provided the necessary guidance and serious advice for my work.

\section{References}

[1] Nazia Parveen, D. S. Venkateswarlu, Implementation of MIMO - OFDM using adaptive multiuser detection in wireless communication" IEEE Trans. Wireless Commun. in 2006, pp. 381-384, Mar. 2012.

[2] L. Dai, Z.Wang, and Z. Yang, "Compressive sensing based timing domain synchronous OFDM transmission for vehicular communications,"IEEE J. Selected Areas in Communications, vol. 31, no. 9,pp. 460-469, Sept. 2013.

[3] S. Vakil and B. Liang, "Cooperative diversity in interference limited wireless networks,"IEEE Trans. Wireless Commun., vol. 7, no.8, pp. 3185-3195, Aug. 2008.

[4] Zhen Gao, Chao Zhang, Zhaocheng Wang, Sheng Chen, "Priori-Information Aided Iterative Hard Threshold: A Low-Complexity High Accuracy Compressive Sensing Based Channel Estimation for TDS-OFDM ,'IEEE Trans. Wireless Commun., Nov. 2015.

[5] A. V. Zelst and T. C. W. Schenk, "Implementation of a Mimo OFDM Based Wireless Lan System ,'IEEE Trans. Signal Processing, vol. 52, no. 2, Feb. 2004, pp. 483-494.

[6] www.mathworks.in

\section{Author Profile}

Jancy Wilson received the B-Tech degree in Electronics and Communication Engineering from Kerala University, Kerala at Baselious Mathews Second College Of Engineering in 2011. And now she is pursuing her $\mathrm{M}$-Tech degree in Communication Engineering under M. G. university in Sree Buddha college of Engineering for women.

Aravind S., working as Assistant Professor in department of Electronics and Communication, Sree Buddha college of Engineering for women, Elavumthitta, Pathanamthitta. 